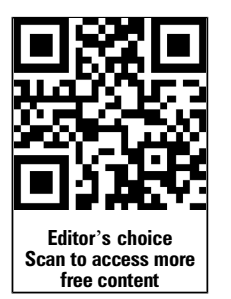

${ }^{1}$ Division of Gastroenterology and Hepatology, Clinical Enteric Neuroscience Translational and Epidemiological Research (C.E.N.T.E.R.), Mayo Clinic College of Medicine, Rochester, Minnesota, USA ${ }^{2}$ Division of Neuroradiology, Department of Radiology, Mayo Clinic College of Medicine, Rochester, Minnesota, USA

\section{Correspondence to}

Professor Michael Camilleri, Division of Gastroenterology and Hepatology, Clinical Enteric Neuroscience Translational and Epidemiological Research (C.E.N.T.E.R.), Mayo Clinic, Charlton 8-110, 200 First St. S.W., Rochester, MN 55905, USA; camilleri. michael@mayo.edu

Received 8 October 2013 Revised 11 December 2013 Accepted 13 December 2013 Published Online First 8 January 2014

\title{
Recent advances in clinical practice challenges and opportunities in the management of obesity
}

\author{
Andres Acosta, ${ }^{1}$ Barham K Abu Dayyeh, ${ }^{1}$ John D Port, ${ }^{2}$ Michael Camilleri ${ }^{1}$
}

\begin{abstract}
Despite advances in understanding the roles of adiposity, food intake, Gl and adipocyte-related hormones, inflammatory mediators, the gut-brain axis and the hypothalamic nervous system in the pathophysiology of obesity, the effects of different therapeutic interventions on those pathophysiological mechanisms are controversial. There are still no low-cost, safe, effective treatments for obesity and its complications. Currently, bariatric surgical approaches targeting the GI tract are more effective than non-surgical approaches in inducing weight reduction and resolving obesity-related comorbidities. However, current guidelines emphasise non-surgical approaches through lifestyle modification and medications to achieve slow weight loss, which is not usually sustained and may be associated with medication-related side effects. This review analyses current central, peripheral or hormonal targets to treat obesity and addresses challenges and opportunities to develop novel approaches for obesity.
\end{abstract}

\section{INTRODUCTION}

The search for effective treatments for obesity has led to a greater understanding of adiposity, GI and adipocyte-related hormones, inflammatory mediators, the gut-brain axis and the hypothalamic nervous system involved in appetite regulation (figure 1). The effects of different interventions on the pathophysiological mechanisms of obesity are the subject of ongoing research. Understanding the pathophysiological mechanisms of obesity provides an opportunity to develop novel approaches to treat, at relatively low cost and enhanced safety, the ever-expanding population of obese people.

\section{SEARCH STRATEGY AND SELECTION CRITERIA}

References for this review were identified through searches in PubMed for articles published from June 1974 to August 2013 by use of the terms "brain-gut axis", "food intake", "vagus nerve", "GI hormones", "treatment", "guidelines", "bariatric surgery", "gastric sleeve", "sleeve gastrectomy", "biliopancreatic diversion" and "endoscopic weight loss procedures" in combination with the term "obesity". Articles resulting from these searches and relevant references cited in those articles were reviewed. Articles published in English were included.

\section{CURRENT STATE OF OBESITY TREATMENT}

Despite information on complex neural, hormonal, metabolic and inflammatory mechanisms in obesity, bariatric-metabolic surgery is the only current treatment for obesity that is effective in the long term. Lifestyle modification and current pharmacological approaches are generally associated with modest (average $5 \mathrm{~kg}$ ) weight loss that is poorly sustained in a majority of patients. ${ }^{1}$ The paradox between the relatively low efficacy of treatments targeting the complex neurohormonal mechanisms in obesity and the greater efficacy of restrictive or malabsorptive surgery is illustrated by their effects on cardiovascular mortality. The Swedish Obese Subjects Trial is the largest prospective bariatric surgery study with longterm follow-up, and it showed a significant reduction in cardiovascular mortality ${ }^{2}$; in contrast, the Look Ahead Trial, a randomised, controlled, National Institutes of Health (NIH)-sponsored trial in 5145 overweight/obese adults with type 2 diabetes designed to determine the long-term health benefits of weight loss achieved by lifestyle modification, showed no difference in cardiovascular mortality after 9 years of follow-up. ${ }^{3}$

\section{Non-surgical interventions}

The two main non-surgical approaches for the treatment of obesity and related complications are intense lifestyle modification and medications. Guidelines, based on Category A evidence and published by the National Heart, Lung and Blood Institute in 1998, suggest "that a 10 percent reduction in body weight reduces disease risk factors. Weight should be lost at a rate of 1 to 2 pounds per week based on a calorie deficit of 500$1000 \mathrm{kcal} / \mathrm{day}{ }^{4}{ }^{4}$ The guidelines also recommend increased physical activity and pharmacological approach to augment weight loss. This approach has been efficacious in multiple large-scale clinical trials: Diabetes Prevention Program showed that intense lifestyle modification prevented by $58 \%$ the incidence of diabetes when compared with placebo controls. ${ }^{5}$ Similarly, the Look Ahead Trial showed that intense lifestyle intervention resulted in 7\% weight loss and improved diabetes control. ${ }^{6}$

In recent years, somewhat efficacious pharmacological approaches (such as sibutramine and rimonabant) received initial regulatory approval but were subsequently withdrawn from the market because of adverse effects such as depression or hypertension resulting from their central actions on adrenergic, serotonergic or cannabinoid mechanisms. Until recently, the only approved medication was the pancreatic lipase inhibitor, orlistat, which is associated with relatively small changes in weight and GI adverse effects, such as bloating and diarrhoea, which reduce compliance with orlistat intake over the long term. New pharmacological approaches, recently approved by the FDA, decrease appetite and result in weight loss: Lorcaserin (Belviq) is a serotonin $2 \mathrm{c}\left(5-\mathrm{HT}_{2 \mathrm{C}}\right)$ receptor agonist that activates pro-opiomelanocortin (POMC) neurons of the hypothalamic arcuate
To cite: Acosta $A, A b u$ Dayyeh BK, Port JD, et al. Gut 2014;63:687-695. 


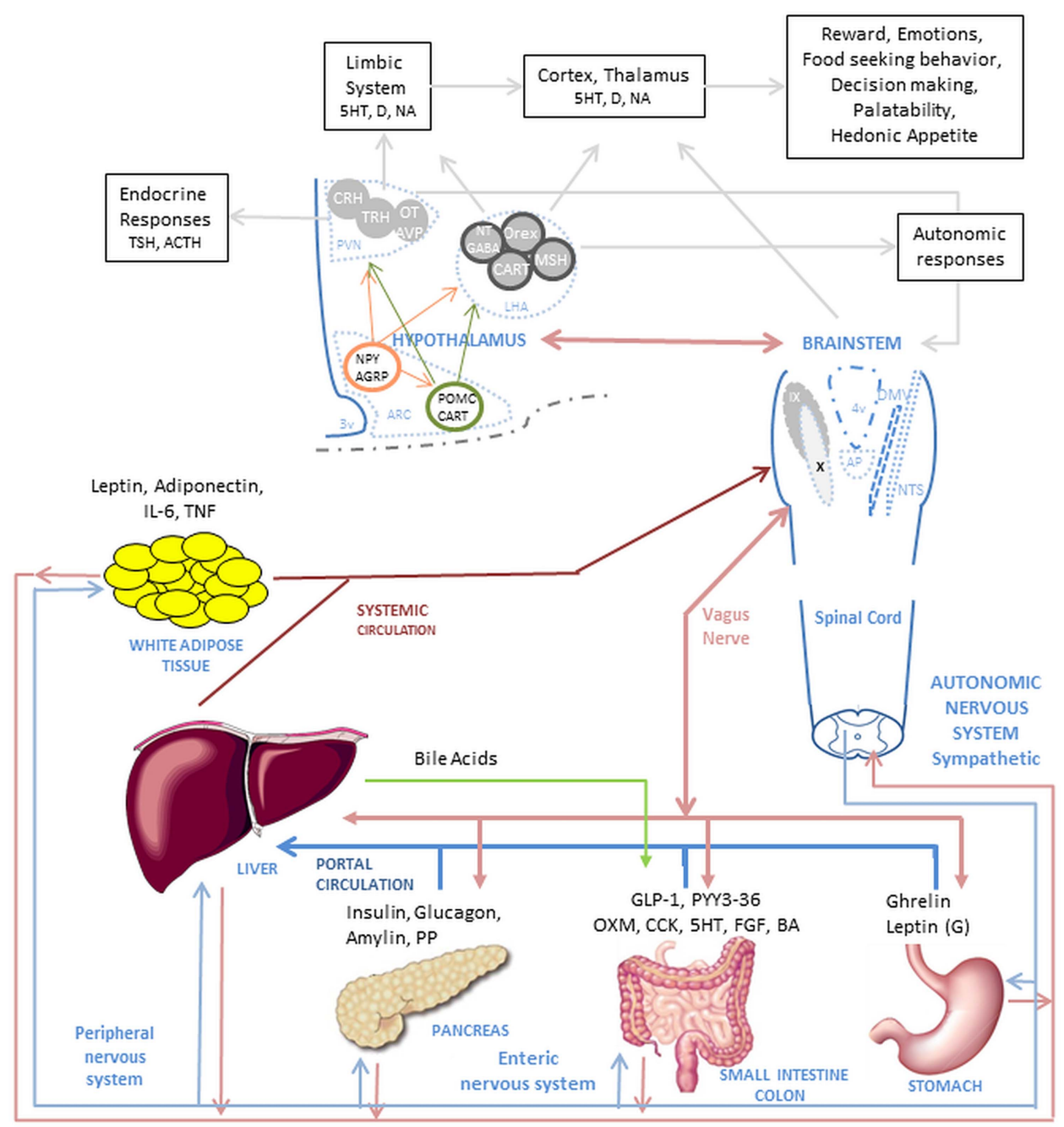

Figure 1 Complex mechanism of food intake regulation. The food intake process initiates when nutrients enter the GI tract. Digestion starts when the nutrients enter the stomach and produce mechanic-dilation decreasing acyl-ghrelin and increasing desacyl-ghrelin and gastric leptin. Stomach dilation sends signals through the vagus nerve and peripheral nervous system to the brainstem and hypothalamus. The digested nutrient passes to the small intestine and colon producing further mechanic-dilation, GI hormones release, bile acid and pancreatic juices secretion. These GI hormones have a local effect (paracrine) and peripheral effect, when secreted into circulation, passed through the liver and affect the muscle, adipose tissue, GI motility and function, and nucleus of the hypothalamus and brainstem. The paracrine and endocrine effect induces satiation and satiety. The muscle and adipose tissue release hormones which affect similar nuclei in the brain. The effect on the hypothalamus and brainstem trigger higher brain area responses, modulating behaviour and enhancing nutrient-related reward. In the hypothalamus, first order neurons in the arcuate nucleus (ARC) modulate appetite by NPYIAGRP pathway and satiation by the POMC/CART pathway. The neurons interact with second order neurons in the Paraventricular nucleus (PVN) and Lateral hypothalamic (LHA) area to send signals to higher brain areas and to the brainstem. In the brainstem, the Nucleus of the tractus solitarius (NST) and dorsal vagal complex (DMNV) interact with the periphery and GI system and brings signals to the higher brain areas and the hypothalamus. Abbreviations in alphabetical order: 5-HT, serotonin; ACTH, adrenocorticotropic hormone; AGRP, agouti-related peptide; ARC, arcuate nucleus; AVP, arginine vasopressin; BA, bile acids; CART, cocaine- and amphetamine-regulated transcript; CCK, cholecystokinin; CRH, corticotropin-releasing hormone; D, dopamine; DMNV, dorsal vagal complex; FGF, fibroblast growth factor -19; GABA, gamma-aminobutyric acid; GLP-1, glucagon-like peptide-1; IL-6, interleukin-6; LHA, lateral hypothalamic; MSH, melanocortin stimulating hormone; NA, noradrenaline; NPY, neuropeptide Y; NST, nucleus of the tractus solitaries; NT, neurotensin; OT, oxytocin; Orex, orexin; OXM, oxyntomodulin; PP, pancreatic polypeptide; PVN, paraventricular nucleus; peptide tyrosine-tyrosine (PYY3)-36, peptide tyrosine-tyrosine 3-36; POMC, proopiomelanocortin; TNF, tumour necrosis factor; TRH, thyroid-releasing hormone; TSH, thyroid-stimulating hormone.

nucleus, decreasing appetite ${ }^{7}$ and resulting in an average $5.8 \%$ weight loss when compared with $2.1 \%$ in the placebo group. ${ }^{8} \mathrm{~A}$ second, approved drug is the combination phenterminetopiramate extended release (ER) (Qsymia) that produces mean 8$10 \%$ weight loss in different trials when compared with $1.6 \%$ weight loss in the placebo group. ${ }^{9}$ This degree of weight loss is lower than that observed with bariatric surgery. In view of the potential for central nervous system (CNS)-mediated and cardiovascular adverse effects with these centrally-acting drugs, they require close postmarketing surveillance. Lorcaserin has high affinity and selectivity and is a full agonist for $5-\mathrm{HT}_{2 \mathrm{C}}$ receptors. By virtue of its high selectivity for $5-\mathrm{HT}_{2 \mathrm{C}}$ receptors relative to $5-\mathrm{HT}_{2 \mathrm{~A}}$ (18-fold) and 5- $\mathrm{HT}_{2 \mathrm{~B}}$ (104-fold) receptors, ${ }^{10}$ lorcaserin avoids induction of hallucinations ${ }^{11}$ and cardiopulmonary toxicity $^{12-14}$ respectively. Nevertheless, the FDA approved phentermine-topiramate ER (Qsymia) with 10 postmarketing requirements. $^{15}$

\section{Bariatric (Metabolic) surgery}

Bariatric surgery remains the most effective treatment option for obese patients. Available procedures include laparoscopic and open Roux-en-Y gastric bypass (RYGB), sleeve gastrectomy, 
adjustable gastric band, vertical banded gastroplasty, duodenal switch and biliopancreatic diversion. RYGB is currently the bariatric surgical procedure of choice. In a meta-analysis of 136 studies including 22094 patients, RYGB resulted in an average excess body weight loss of $62 \%$, with resolution of diabetes in $84 \%$, of hypertension in $68 \%$, and of obstructive sleep apnoea in $81 \%$, and improved hyperlipidaemia in 97\%. ${ }^{16}{ }^{17}$ Unlike medications and lifestyle modifications, the effects of bariatric surgery seem to be sustained in the long term. Thus, the recently updated Swedish Obese Subjects Study demonstrated mean changes in body weight after bariatric surgery (specifically with 13\% RYGB, 19\% gastric banding and 68\% vertical banded gastroplasty) $-23 \%$ (at 2 years), $-17 \%$ (at 10 years), $-16 \%$ (at 15 years) and $-18 \%$ (at 20 years). ${ }^{2}$

Despite proven efficacy and the fact that mortality from bariatric surgery is comparable to that of cholecystectomy or appendectomy in bariatric centres with high surgical volumes, it is estimated that less than $1 \%$ of obese subjects who qualify for bariatric surgery will undergo such intervention. ${ }^{18}$ High costs and early and late complications of bariatric surgery are the main hurdles for widespread use. Early complications include anastomotic leaks, internal hernias, thromboembolic events, bowel obstruction, GI haemorrhage and wound complications. Late complications include gallstones formation, marginal ulceration, anastomotic stricture, incisional hernia, gastro-gastric fistula, dumping syndrome, micronutrient deficiencies and weight regain.

Given the current state of therapies for obesity, it is timely to question the 1998 guidelines that recommend slow weight loss with non-surgical approaches that produce modest and poorly sustained efficacy and potential centrally mediated side effects. The next sections review advances in our understanding of central and peripheral pathways controlling energy intake and expenditure in that order and discuss the potential to develop effective, minimally invasive treatments for obesity.

\section{TARGETING THE CENTRAL NERVOUS SYSTEM IN OBESITY TREATMENT}

Appetite and satiety are controlled by hypothalamic centres in connection with the limbic (emotions and reward) and the autonomic nervous systems. These systems may provide novel targets for development of drug treatment for obesity. There are fenistrations in the blood-brain barrier (BBB) that are limited to specific brain regions, specifically the area postrema in the floor of the fourth ventricle and the subfornical organ and organum vasculosum of the lamina terminalis in the anterior wall of the third ventricle that are highly interconnected with the supraoptic, median preoptic and paraventricular nuclei of the hypothalamus. The latter organs play a role in water homeostasis and associated behaviours (thirst). In contrast, area postrema and the associated nucleus of the solitary tract (NST) and dorsal motor nucleus of the vagus nerve (DMNV) are the more likely locations for GI neuropeptide 'sensing' by the CNS. ${ }^{19}$ Regardless, either the fenistrations or specific transport processes are able to deliver gut or adipose-related hormones to these pivotal brain centres. 1920

\section{The hypothalamic nuclei}

The CNS coordinates a complex and dynamic process to regulate energy balance. Food intake, appetite and satiation are mainly regulated by the gut-brain axis, ${ }^{21} 22$ that is, gut hormones, the vagal complex, brainstem, medial basal hypothalamus and higher cortical brain centres. ${ }^{21}$ The current overarching hypothesis is that appetite is stimulated by the secretion of acyl-ghrelin (AG) from the stomach during the fasting period. Ghrelin acts on specialised neurons in the arcuate nucleus of the hypothalamus to activate the agouti-related peptide/neuropeptide Y (AgRP/NPY) pathway. ${ }^{23-}$ ${ }^{25}$ This pathway is responsible for stimulating appetite centres in the cerebral cortex that are involved in food-seeking behaviour and prepare the GI tract for food intake by a vagal response. ${ }^{26}$ Satiation (or the feeling of fullness after meal ingestion) and satiety (absence of appetite) are induced by several hormones including leptin, insulin, glucagon, cholecystokinin, peptide YY, oxyntomodulin (OXM) and glucagon-like peptide-1 (GLP-1), which are secreted from the adipose tissue, pancreas and GI tract after food intake. These gut hormones inhibit the AgRP/ NPY pathway in the arcuate nucleus and stimulate the POMC/ $\alpha$-melanocyte stimulation hormone ( $\alpha \mathrm{MSH}$ ) pathway. ${ }^{22} 27$ The neurons in the arcuate nucleus are called 'first-order' neurons because of their 'direct' contact with peripheral hormones that mediate satiation or satiety. 'Second-order' neurons arise from the paraventricular nucleus, lateral hypothalamic area and ventromedial nucleus of the hypothalamus. ${ }^{28}$ Through projections caudally to the NST and DMNV in the brainstem, these second order neurons stimulate the gastric vagal efferent fibres to delay gastric emptying by stimulating intra-gastric nitric oxide system (NOS) neurons, thus decreasing motility. ${ }^{29} 30$ Second-order neurons also increase energy expenditure by inducing hyperthermia, ${ }^{31} 32$ and cranially directed neurons stimulate higher brain centres and produce food reward or feeling of wellbeing after a meal. ${ }^{30}$ 32-36 These neuronal circuits ultimately control energy intake and expenditure by interacting with the GI tract, peripheral nervous system and adipose tissue.

Current understanding of the role of the hypothalamus and its circuits in energy balance is based on in vitro and in vivo observations of the effects of hypothalamic injuries or knock down, knock out and/or overexpression of target genes or pathways. However, interpretation of these experimental models is complicated by the presence of redundant pathways or compensatory mechanisms that can adapt the response to modification of specific targets, receptors or mechanisms. Novel techniques applied in animal models, such as optogenetics (that can photostimulate specific neurons) or short hairpin RNA studies (that can 'silence' one or more specific gene targets), may provide more comprehensive insights of the control of appetite and satiation without evoking compensatory mechanisms. The application of such techniques on AgRP neurons showed this pathway can regulate feeding behaviour independent of the POMC pathway, ${ }^{37}$ challenging the current paradigm of interaction of the AgRP/NPY and the POMC/ $/ \mathrm{MSH}$ pathways and questioning accuracy of current knowledge based on genetically modified animal models. ${ }^{38}$

In humans, less invasive methods have been developed to study these hypothalamic nuclei. Ideally, one would like to measure the electrical activity (action potentials) in these nuclei before, during and after eating to determine their signalling mechanisms. Unfortunately, this is not possible with current techniques. A surrogate measure of neural activity is blood flow, specifically, as a brain area becomes electrically active, blood flow increases to supply oxygen and glucose and remove carbon dioxide.

Brain blood flow can be measured using MRI. Three general imaging techniques are available for measuring blood flow to the hypothalamus, each with their own strengths and limitations. The primary challenge for each of these techniques is the small size of the hypothalamic nuclei; for example, each half of the hypothalamus is only $3-4 \mathrm{~mm}$ thick, and the arcuate 
nucleus is a cell cluster within the hypothalamus measuring only a few hundred microns in diameter. MRI systems with higher magnetic field strengths (eg, 3 tesla $(\mathrm{T}), 7 \mathrm{~T}$ ) are more beneficial for imaging the hypothalamus as they have the higher resolution necessary to accurately measure such small structures.

Contrast-enhanced perfusion imaging is the most common MR technique to measure blood flow and has the highest resolution. However, this technique requires an intravenous bolus injection of gadolinium contrast and, therefore, can only be performed once per day due to potential renal toxicity. 'Functional MRI' is a technique that measures relative changes in blood flow between two different alternating experimental conditions. However, this technique is better suited to experimental designs where the two conditions are varied relatively rapidly (on the timeframe of seconds); most studies of hunger and satiety occur in a timeframe of minutes and cannot easily alternate between different conditions. Furthermore, this technique is susceptible to artefacts from the sphenoid and ethmoid sinuses that limit evaluation of the hypothalamus.

Pulsed arterial spin labelling (PASL) is an MR technique that labels blood with a magnetic 'tag' as it enters the skull and images it in the brain a short time later. PASL is ideally suited for the study of the hypothalamus as it: (1) does not require intravenous contrast and therefore can be performed many times over a few hour period; (2) does not suffer from significant susceptibility artefact; (3) yields highly reliable and reproducible quantifiable measurements of blood flow; and (4) using a 3 T MRI scanner, has sufficient resolution to measure global hypothalamic blood flow while minimising the influence of neighbouring areas.

As it is a relatively new MRI technique, only a few PASL studies of obesity have been performed to date. For example, PASL was used to measure absolute blood flow changes in different regions of the brain in response to stimuli such as ingestion of different simple sugars; thus, there is differential stimulation of hypothalamic regions with glucose and fructose. ${ }^{39-41}$ This approach has the potential to understand the activation of anatomical regions of the hypothalamus in response to nutrients during postprandial satiation.

Further studies are required to understand the adaptive and complex processes and neuroplasticity in the hypothalamus of obese subjects and to explain the function of CNS centres in the weight gain in already obese subjects in a food-rich situation. Specifically, it is still unclear why the brain functions as though the individual is still 'hungry' despite the presence of obesity, or why it is so difficult to maintain weight loss after a period of food-deprivation. ${ }^{42}{ }^{43}$ Some authors have claimed that the longterm effect of bariatric surgery is mediated by a change in hypothalamic plasticity and resetting of the homeostatic 'set point', that is, the hypothalamic adaptive response that sets the total caloric intake per day. ${ }^{44}$ Experimental data supporting such a set point are changes in hypothalamic activity after bariatric surgery in diet-induced obesity in rodents ${ }^{45}$; in contrast, other studies refute the presence of a set point since no changes in the hypothalamus were observed after bariatric surgery when compared with caloric restricted rodents. ${ }^{46-48}$

With the introduction of MRI with PASL, it may be possible to clarify whether there are changes in the hypothalamic activity in humans during different stages of the postprandial period (cephalic, gastric and enteric) as well as in response to pharmacological, behavioural or surgical interventions.

The continuing challenge is to develop CNS targeted therapies that avoid effects on pathways unrelated to energy intake in the higher brain centres that may serve other physiological and psychological functions. Using novel imaging technologies, there is potential to appraise the responsiveness of specific hypothalamic and other brainstem and brain centres to macronutrients, a nutrient-poor or nutrient-rich environment, and to therapy directed at these redundant and complex pathways.

\section{The brainstem-hypothalamic connections}

The brain stem, through the predominantly afferent nucleus of the NST and the integrative and DMNV complex, controls the enteric nervous system in response to signals from energy levels in the periphery. ${ }^{26}{ }^{30}$ The energy levels also influence brainstem nuclei in the area postrema in the floor of the fourth ventricle where there is a thin BBB. ${ }^{49}$ The DMNV has ascending and descending connections with the arcuate, paraventricular nucleus and lateral area of the hypothalamus and other higher brain centres to regulate food intake. ${ }^{30}$

Like the hypothalamus, the brainstem plays a major role in mediating brain-gut coordination through the nuclei mentioned above. As with the hypothalamus, the DMNV has a permeable BBB and is susceptible to the influence of circulating hormones such as incretins and glucose-regulatory hormones that may be associated with taste aversion in animal models and nausea in humans through activation of the area postrema in the brainstem. ${ }^{50}$

\section{The role of the vagus nerve}

The vagus nerve innervates most of the regions of the GI tract involved in energy intake, satiation and digestion, ${ }^{30}$ and it serves as a crucial link between the brain, brainstem and gut.

The afferent neuronal fibres of the ventral and dorsal vagal trunks in the abdomen are involved in regulating appetite and satiation. The vagus nerve is directly stimulated by mechanical stretch or change in viscus tension when food, or later chyme, passes through the lumen of the GI tract. The vagus nerve is also stimulated indirectly by neurohormonal mechanisms activated by the same mechanical and chemical stimuli arising from the gut. In the stomach, ghrelin secretion inhibits afferent vagal fibres to induce appetite-an orexigenic effect. Leptin secreted in the stomach stimulates vagal fibres and induces satiety-an anorexigenic effect. In the rest of the GI transit of chyme, the vagal afferent fibres are stimulated by other anorexigenic hormones such as cholecystokinin (CCK), GLP-1 or peptide YY.

Efferent vagal neuronal fibres control much of the motor and secretory apparatus of the digestive tract involved in food digestion and absorption. Partial vagotomy, or total subdiaphragmatic vagotomy, or intermittent vagal nerve electrical stimulation to inhibit vagal function in humans ${ }^{51}$ decreased food intake and induced early satiety and weight loss.

The vagus nerve plays a dual role, interacting with anorexigenic and orexigenic pathways. These interactions appear to be altered in obesity. ${ }^{52}$ Greater understanding of methods to modulate vagal activity to induce weight loss could conceivably arise through stimulation of the anorexigenic pathway or inhibition of the orexigenic pathway. Clearly, further studies are needed to understand the effect of the vagus nerve in weight loss and glycemic control.

\section{TARGETING THE GUT IN THE TREATMENT OF OBESITY Bariatric surgery}

Bariatric surgery, especially RYGB, has thus far proved to be the most effective intervention for obesity and type 2 diabetes. The exact mechanism by which RYGB exerts its effects on weight loss and glycemic regulations is not fully understood; the timing of resolution of diabetes suggests that RYGB's effects on 
diabetes are independent of the degree of weight loss. Animal and human studies suggest that the restriction of food reservoir and malabsorption is insufficient to account for RYGB's effects to enhance satiation and satiety, improve food seeking behaviours and food selection, increase energy expenditure and improve glucose homeostasis. ${ }^{53-55}$ Thus, further studies are required to clarify physiological alterations in gut neuroendocrine signalling, GI motility, autonomic nervous system signalling, bile acid production and absorption, gut microbiota and other potential interactions with the CNS, pancreas, liver, muscles and adipose tissue in response to RYGB.

RYGB surgery is a complex procedure with at least five distinct components, all of which may have biological relevance in the induction of weight loss or ameliorating hyperglycaemia: (1) isolation of the gastric cardia by creation of a small gastric pouch, (2) exclusion of the distal stomach from contact with food, (3) exclusion of the proximal intestine from contact with food, (4) exposure of the jejunum to partially digested nutrients and (5) partial vagotomy (figure 2). Each of these distinct components of RYGB has the potential to alter appetite, energy absorption and expenditure, and glucose homeostasis; thus, replication of these individual anatomic alterations can be novel approaches to develop peripherally targeted treatments for obesity. The next section analyses each of these distinct components.

\section{The gastric cardia and ghrelin}

Isolation of the gastric cardia and exclusion of the distal stomach may initially limit caloric intake by induction of nausea (and rarely vomiting), thereby discouraging overeating. In addition, the stimulation of the gastric mechanical and chemical receptors, rapid pouch emptying and release of ghrelin may also contribute to the induction of weight loss. ${ }^{56} 57$ Ghrelin is a peptide produced from the pre-pro ghrelin gene, mainly in the stomach. It is known to increase gastric emptying and to

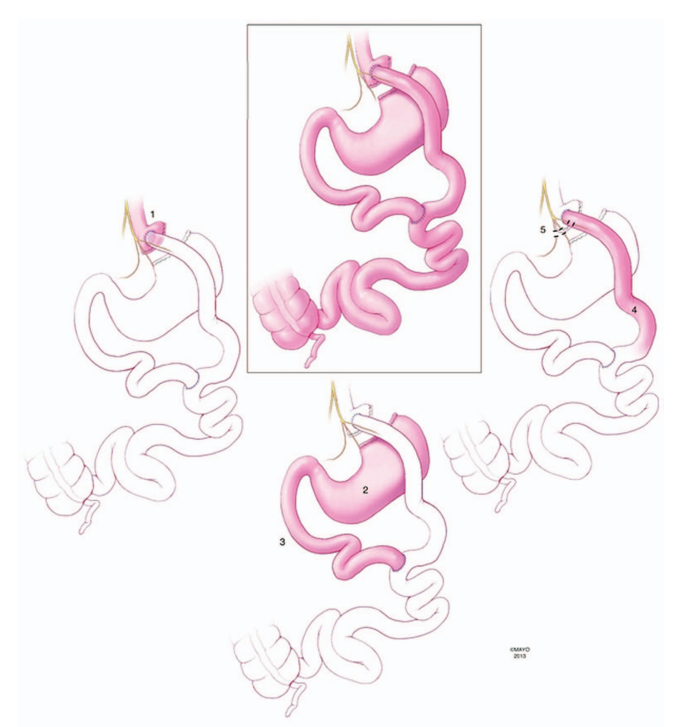

Figure 2 Biological relevance of Roux-en-Y gastric bypass (RYGB) surgery. RYGB is a complex procedure with at least five distinct components, all of which may have biological relevance in the induction of weight loss or ameliorating hyperglycaemia: (1) isolation of the gastric cardia by creation of a small gastric pouch, (2) exclusion of the distal stomach from contact with food, (3) exclusion of the proximal intestine from contact with food, (4) exposure of the jejunum to partially digested nutrients (5) and partial vagotomy. undergo cyclical changes in blood concentrations in the fasting and postprandial periods, being highest during fasting. AG is metabolised by the ghrelin activating enzyme, ghrelin-O-acyltransferase (GOAT), to deacyl-ghrelin (DAG). AG and DAG have different physiological effects: $A G$ increases gastric emptying and appetite, whereas DAG decreases gastric emptying, induces postprandial fullness, and improves insulin sensitivity. ${ }^{58}$ The published literature suggests there is a decrease in plasma ghrelin post-RYGB, with attenuation of the cyclical changes in ghrelin levels. ${ }^{59} 60$ The literature is unclear about effects of AG or its metabolite, DAG, whose actions are different, as detailed above. Thus, the contribution of the changes in plasma ghrelin concentrations to the clinical efficacy of RYGB requires further investigation.

\section{Bypass of the proximal small intestine}

The proximal small intestine is extremely efficient in absorption of monosaccharides, ${ }^{61}$ and it plays a major role in glucose homeostasis and in the pathogenesis of diet-induced diabetes. Wang and colleagues have shown that direct administration of lipids into the upper intestine of non-diabetic rats suppressed glucose production through an intestine-brain-liver neural axis; this effect was not observed in rats with diet-induced diabetes. ${ }^{62}$ Furthermore, Goto-Kakizaki (GK) type 2 diabetic rats that undergo duodeno-jejunal bypass (DJB) develop markedly improved oral glucose tolerance compared with rats that undergo gastrojejunostomy (which does not bypass the duodenum); these effects are reversed with re-establishing flow through the duodenum. ${ }^{63}$

Patients who undergo DJB have greatly improved glycemic control with only minimal effect on body weight. ${ }^{64}{ }^{65}$ However, diabetes remission rates are much lower after DJB compared with traditional RYGB, suggesting that other effects of RYGB are important in weight loss and that diabetes remission rates may be proportional to the degree of weight loss. ${ }^{66}$ On the other hand, vertical sleeve gastrectomy (VSG) does not bypass the duodenum and produces significant weight loss and glycemic improvement. In a randomised controlled trial, VSG was associated with weight loss comparable to that observed with RYGB. ${ }^{67}$ The incretins and insulin-glucose responses were also similar 3 months after the procedures, although RYGB had a greater response than VSG earlier than 3 months postsurgery. ${ }^{68}$ The potential additional role of the bypassed duodenum in ameliorating glycemic control, independent of the effects of incretins, is supported by a recent meta-analysis which showed that RYGB surgery produced a higher rate of remission of type 2 diabetes when compared with VSG (OR 2.46 CI 1.48 to 40.9) ${ }^{69-72}$ Further studies are needed to understand the differences in these bariatric surgeries and to ascertain if the glycemic improvement is due to weight loss alone or whether the bypass of the duodenum is really necessary.

\section{Incretins}

Within the mucosa of the small intestine, enteroendocrine cells express functional gustducin-coupled sweet taste receptors that 'taste' luminal nutrients and release gut peptides that mediate satiety and enhance insulin secretion (incretins). ${ }^{73}$ These peptides exert their effects through interaction with the afferent vagal and spinal nerve fibres innervating the gut, or by entering the blood stream to function as hormones, influencing the area postrema or hypothalamic areas through the locally permeable BBB.

GLP-1 is the major known incretin, synthesised from the enteroglucagon or pre-proglucagon gene in the enteroendocrine 
L-cells, which are located in the proximal small bowel, but are more abundant in the distal small bowel and colon. ${ }^{75}$ In addition to GLP-1, the enteroglucagon gene encodes multiple peptides involved in energy balance and glycemic control; these include glucagon and OXM.

GLP-1 is released in response to contact with luminal nutrients or bile acids, and by submucosal neural stimulation mediated by voltage-gated $\mathrm{Ca}^{++}$channels. $^{76}$ GLP-1 inhibits glucagon secretion, stimulates glucose-dependent insulin secretion, increases insulin sensitivity, induces nausea and satiety and is associated with weight loss. ${ }^{77-79}$ GLP-1 levels are blunted in response to luminal glucose in type 2 diabetes, resulting in an early defective insulin response to the luminal glucose load. ${ }^{80} 81$

GLP-1 and insulin levels significantly increase in the early postprandial period after RYGB, an effect that is not observed with laparoscopic adjustable gastric band or diet-induced weight loss because the latter do not accelerate gastric emptying of nutrients. ${ }^{82} 83$ The early postprandial increase in these peptide levels results from enhanced delivery of partially digested nutrients to the distal small intestine, directly stimulating the L cells. Indeed, isoperistaltic ileal transposition above the ligament of Treitz (which accelerates delivery of nutrients to the distal small bowel) in diabetic rats and humans resulted in significant improvement in GLP-1 production in response to glucose and improved glucose homeostasis. ${ }^{84-86}$ The endogenous GLP-1 effect on glucose homeostasis and GI motility is sustained when examined an average of 5 years after RYGB. ${ }^{87}$ In a randomised, controlled trial, the GLP-1 agonist, liraglutide, $3 \mathrm{mg}$, produced a significant mean weight loss of $7.2 \mathrm{~kg}$ compared with $2.8 \mathrm{~kg}$ in the placebo group. ${ }^{88}$ Conversely, studies in rodents have shown that the effect of weight loss and glycemic improvement after VSG is independent of GLP-1 activity. ${ }^{89}$

Peptide tyrosine-tyrosine (PYY) is another gut hormone with significant effect on the ileal brake and regulation of food intake. Pharmacological doses of PYY induced satiety; ${ }^{90}{ }^{91}$ PYY is also increased after $\mathrm{RYGB}^{92} \mathrm{~A}$ large phase II clinical trial of intranasal peptide $\mathrm{YY}_{3-36}$ had to be discontinued early due to severe nausea and vomiting, ${ }^{93}$ which is most likely explained by PYY stimulating the area postrema in the brainstem resulting in nausea $^{50}$ or delay in gastric emptying induced by $\mathrm{PYY}_{3-36 .}{ }^{94-96}$

Quantifying the contribution of the rise in GLP-1 and other incretins to the therapeutic effects of RYGB and weightindependent improvement in glycemic control requires further study. While further information may lead to novel applications of incretins in weight loss therapy, experience with GLP-1 and intranasal $\mathrm{PYY}_{3-36}$ suggests that adverse effects such as nausea may limit the potential clinical efficacy.

\section{Autonomic denervation in RYGB}

The vagus nerve enters the abdomen from the oesophageal plexus as anterior and posterior nerve trunks. Denervation of sub-diaphragmatic branches of these trunks may occur after RYGB. The contribution of this denervation to weight loss and metabolic changes after RYGB is unclear.

Through the actions of melanocortin- 4 receptors (MC4R) in the DMNV and thoracic sympathetic preganglionic neurons, the autonomic nervous system plays an important role in the improved insulin sensitivity and enhanced energy expenditure after RYGB. ${ }^{97}$ Furthermore, a variant in the human $M C 4 R$ $(\mathrm{I} 215 \mathrm{~L})$ locus that enhances basal activity of the receptor is associated with improved outcomes after RYGB. The peripheral signals mediating enhanced activation of central MC4R signalling are yet to be identified. ${ }^{98}$ Further studies are needed to understand the role of MC4R signalling in satiety.

\section{Microbiome in RYGB}

Emerging evidence suggests that microbes that reside in the human GI tract can contribute to development of obesity. As a dynamic ecosystem, the microbiota closely interacts with host metabolism, for example, by breaking down otherwise indigestible carbohydrates and increasing short chain fatty acid absorption in the colon. This provides the host with additional energy and increases fat storage in adipose tissue. ${ }^{100} 101$ The microbiota composition in obese individuals appears to be structurally and functionally different from that of lean individuals, and it appears to be adapted to more efficient energy extraction and storage, contributing to obesity and its metabolic consequences. This adaptation is termed 'obesity associated dysbiosis' ${ }^{102}$

Recently, RYGB has been shown to cause shifts in faecal microbiota profiles, potentially contributing to some of the favourable effects of RYGB. ${ }^{103-105}$ In mice, changes in gut microbiota after RYGB seem to be mediated by anatomical rearrangement of the GI tract, rather than being a response to food restriction mediated weightloss. ${ }^{105}$ However, these findings have not been replicated in humans. It is also unclear which anatomical or functional component of the RYGB is predominantly responsible for the change in gut microbiota; the potential candidate mechanisms include changes in gastric $\mathrm{pH}$, bile acid production and circulation, GI tract motility, gut hormonal profiles, and gut-brain interaction, each of which can independently result in a change in the gut microbiota. ${ }^{106}$

In summary, the effects of bariatric surgery are fundamentally different from those of restrictive diets. The latter often lead to physiological responses to weight loss that actually increase hunger and decrease energy expenditure, resulting in regain of the lost weight. ${ }^{199}$ The long-term efficacy of RYGB provides a paradigm shift in our understanding of the physiological mechanisms of reduced appetite and weight loss and the physiological responses which differ from those operant in diet-induced weight loss. The success of RYGB encourages focus on therapeutic approaches in obesity directed at the GI tract.

\section{PERIPHERAL INTERVENTIONS AS ALTERNATIVES TO BARIATRIC SURGERY}

The previous discussion suggests five anatomical alterations may mediate the effects of RYGB on energy intake and expenditure. It also suggests that a multitude of physiological adaptations in the homeostatic mechanisms involved in body weight regulation may need to be addressed to overcome the compensatory responses leading to weight regain seen with restrictive diets.

Emerging technologies have opened the door to the use of approaches and devices to reproduce endoscopically many of the anatomical alterations of bariatric surgery. Targeting peripheral mechanisms has the potential to provide effective treatment of obesity and its associated disorders in a cost-effective and minimally invasive alternative to traditional bariatric surgery. This approach may also be applied in those with moderate obesity, vulnerable populations, such as children and adolescents, and at-risk superobese individuals. ${ }^{107}$

Endoscopic implantation of duodenal-jejunal bypass sleeve made from a Teflon liner (EndoBarrier, GI Dynamics, Lexington, Massachusetts, USA) shows promise and efficacy in the management of obesity and associated diabetes. ${ }^{108} 109$ When deployed in the duodenal bulb, this impermeable fluoropolymer sleeve, extending $60 \mathrm{~cm}$ into the small bowel, creates a mechanical barrier that allows food to bypass the duodenum and proximal jejunum, thus potentially manipulating the enteroinsular system (figure 3). Several prior studies have 


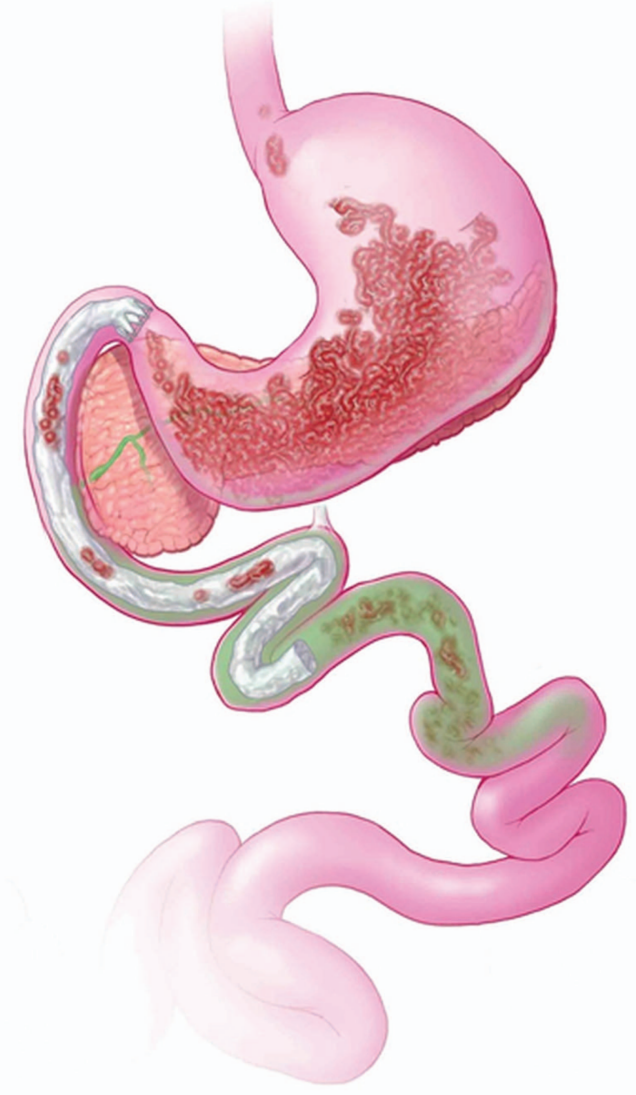

Figure 3 Duodenal-jejunal bypass sleeve. The duodenal-jejunal bypass sleeve deploys in the duodenal bulb, this impermeable fluoropolymer sleeve extending $60 \mathrm{~cm}$ into the small bowel creates a mechanical barrier that allows food to bypass the duodenum and proximal jejunum, thus potentially manipulating the enteroinsular system (EndoBarrier, GI Dynamics, Lexington, Massachusetts, USA).

documented the technique's feasibility and efficacy on weight loss and on comorbidity such as non-alcoholic fatty liver disease. ${ }^{108-112}$ Recent rodent studies showed that the diversion of nutrients away from the duodenum decreases food intake, improves glucose homeostasis, increases circulating bile acids and increases small intestine villus length ${ }^{113}$ A pivotal U.S. multicentre FDA registry trial for this device is currently underway. The impact of this device in the long term requires elucidation as insulin sensitivity improved by $>50 \%$ as early as 1 week after implantation, but there was a trend towards deterioration in all the above-mentioned variables 26 weeks after explantation. ${ }^{114}$

Recent advances in flexible endoluminal endoscopy now provide the necessary endoscopic suturing tools for transoral endoscopic gastric volume reduction in a fashion similar to sleeve gastrectomy. The feasibility and short-term efficacy of an endoscopic sleeve gastroplasty for the treatment of obesity has been recently reported. ${ }^{115}$

\section{CONCLUSIONS}

The obesity epidemic requires concerted effort, including behavioural, societal, legislative and therapeutic interventions. We have focused on the therapeutic interventions and identified challenges and opportunities to impact the unmet needs with non-surgical interventions (table 1). Despite the impact of bariatric surgery, we believe that further insights on vagal, hypothalamic and GI hormonal control are key to the development of interventions that can be applied in the large number of eligible patients with obesity and metabolic syndrome. It is also conceivable that future interventions targeting the microbiome or intraluminal mechanisms such as interference with intraluminal digestion or bile acid-related augmentation of incretin responses (not discussed here) may be feasible, peripherally-directed therapies. Meanwhile, the search for the magic bullet in central control mechanisms will continue, though it remains paradoxical that, despite all the drug development programmes being centred on hypothalamus or central mechanisms controlling appetite, the peripheral targets of bariatric surgery constitute the most salient direction for practical and effective obesity treatment at present.

Acknowledgements We thank Cindy Stanislav for secretarial assistance.

Contributions $A A, B K A-D$ and $M C$ were responsible for study conceptualisation and writing of manuscript. JDP was responsible for writing of manuscript.

Funding National Institutes of Health to MC for studies in obesity (NIH RO1DK67071).

Table 1 Paradox between bariatric (RYGB) surgery and pharmacological interventions for obesity

\begin{tabular}{|c|c|c|}
\hline & RYGB surgery & Pharmacological interventions \\
\hline Body weight loss & Up to $33 \%$ & Up to $10 \%$ \\
\hline Body weight loss maintenance & Sustained loss of up to $18 \%$ of BW over 15 years & Poorly sustained over 2 years \\
\hline Rate of weight loss & Rapid within first year & Slow-steady \\
\hline Reduce cardiovascular mortality & Yes & Unproven to date; studies ongoing \\
\hline Insulin resistance & Improvement to resolution of insulin resistance & No effect \\
\hline Dyslipidaemia & Mild improvement & No change \\
\hline Hypertension & Significant improvement & Mild improvement \\
\hline \multicolumn{3}{|l|}{ Mechanism of action } \\
\hline Hypothalamus & Questionable effect & $\begin{array}{l}\text { L: Increase POMC pathway via } 5 \mathrm{HT} 2 \mathrm{c} \text { receptor } \\
\text { P-T: noradrenergic—dopaminergic pathways; unclear mechanism }\end{array}$ \\
\hline Brainstem & Unclear & No effect \\
\hline Vagus nerve & Cuts gastric branches of vagus nerve & No effect \\
\hline GI hormones & Increase GLP-1, PP, PYY, OXM (especially early postprandially) & No effect \\
\hline Effective gastric reservoir size & Decreased & No effect \\
\hline Side effects & Mainly surgery related nutrients deficiency & Lorcaserin and Topiramate-Phentermine (ER) may have CNS side effects \\
\hline
\end{tabular}




\section{Competing interests None.}

Provenance and peer review Not commissioned; externally peer reviewed.

\section{REFERENCES}

1 Anderson JW, Konz EC, Frederich RC, et al. Long-term weight-loss maintenance: a meta-analysis of US studies. Am J Clin Nutr 2001;74:579-84.

2 Sjöström L. Review of the key results from the Swedish Obese Subjects (SOS) trial -a prospective controlled intervention study of bariatric surgery. J Intern Med 2013:273:219-34

3 Cardiovascular Effects of Intensive Lifestyle Intervention in Type 2 Diabetes. N Engl J Med 2013:369:145-54.

4 Pi-Sunyer FX, Becker DM, Bouchard C, et al. Clinical guidelines on the identification, evaluation, and treatment of overweight and obesity in adults: Executive summary. Am J Clin Nutr 1998;68:899-917.

5 Knowler WC, Barrett-Connor E, Fowler SE, et al. Reduction in the incidence of type 2 diabetes with lifestyle intervention or metformin. N Engl J Med 2002;346:393-403.

6 Pi-Sunyer X, Blackburn G, Brancati FL, et al. Reduction in weight and cardiovascular disease risk factors in individuals with type 2 diabetes: one-year results of the look AHEAD trial. Diabetes Care 2007:30:1374-83.

7 Sohn JW, Xu Y, Jones JE, et al. Serotonin 2C receptor activates a distinct population of arcuate pro-opiomelanocortin neurons via TRPC channels. Neuron 2011;71:488-97.

8 Smith S, Weissman N, Anderson C, et al. Multicenter, placebo-controlled trial of lorcaserin for weight management. N Engl J Med 2010;363:245-56.

9 Allison D, Gadde K, Garvey W, et al. Controlled-release phentermine/topiramate in severely obese adults: a randomized controlled trial (EQUIP). Obesity (Silver Spring, Md) 2012:20:330-42.

10 Thomsen W, Grottick A, Menzaghi F, et al. Lorcaserin, a novel selective human 5-hydroxytryptamine $2 \mathrm{C}$ agonist: in vitro and in vivo pharmacological characterization. J Pharmacol Exp Ther 2008;325:577-87.

11 Nichols D. Hallucinogens. Pharmacol Ther 2004;101:131-81.

12 Devereux R. Appetite suppressants and valvular heart disease. N Eng/ J Med 1998;339:765-6.

13 Fitzgerald LW, Burn TC, Brown BS, et al. Possible role of valvular serotonin 5-H (2B) receptors in the cardiopathy associated with fenfluramine. Mol Pharmacol 2000;57:75-81.

14 Launay JM, Hervé $P$, Peoc'h $K$, et al. Function of the serotonin 5 -hydroxytryptamine $2 \mathrm{~B}$ receptor in pulmonary hypertension. Nat Med 2002;8:1129-35.

15 FDA approves weight-management drug Qsymia. In: Administration USFaD, ed. 2012.

16 Buchwald H, Williams SE. Bariatric surgery worldwide 2003. Obes Surg 2004;14:1157-64.

17 Buchwald H, Oien DM. Metabolic/bariatric surgery Worldwide 2008. Obes Surg 2009:19:1605-11.

18 Buchwald H, Oien D. Metabolic/Bariatric surgery worldwide 2011. Obes Surg 2013:23:427-36

19 Banks W. The blood-brain barrier: connecting the gut and the brain. Regul Pept 2008;149:11-14.

20 Hileman S, Pierroz D, Masuzaki H, et al. Characterizaton of short isoforms of the leptin receptor in rat cerebral microvessels and of brain uptake of leptin in mouse models of obesity. Endocrinology 2002;143:775-83.

21 Lenard NR, Berthoud H-R. Central and Peripheral Regulation of Food Intake and Physical Activity: Pathways and Genes. Obesity 2008;16:S11-22.

22 Gardiner JV, Jayasena CN, Bloom SR. Gut hormones: a weight off your mind. J Neuroendocrinol 2008:20:834-41.

23 Baynes KC, Dhillo WS, Bloom SR. Regulation of food intake by gastrointestinal hormones. Curr Opin Gastroenterol 2006;22:626-31.

24 Cone RD, Cowley MA, Butler AA, et al. The arcuate nucleus as a conduit for diverse signals relevant to energy homeostasis. Int J Obes Relat Metab Disord 2001;25(Suppl 5):S63-7.

25 Cowley MA. Hypothalamic melanocortin neurons integrate signals of energy state. Eur J Pharmacol 2003:480:3-11.

26 Grill HJ. Distributed neural control of energy balance: contributions from hindbrain and hypothalamus. Obesity (Silver Spring) 2006;14(Suppl 5):216S-21S.

27 Hillebrand JJ, de Wied D, Adan RA. Neuropeptides, food intake and body weight regulation: a hypothalamic focus. Peptides 2002;23:2283-306.

28 Heijboer AC, Pijl H, Van den Hoek AM, et al. Gut-brain axis: regulation of glucose metabolism. J Neuroendocrinol 2006;18:883-94.

29 Smith G. The direct and indirect controls of meal size. Neurosci Biobehav Rev 1996:20:41-6.

30 Berthoud H-R. The vagus nerve, food intake and obesity. Regul Pept 2008;149:15-25.

31 Azzara A, Sokolnicki J, Schwartz G. Central melanocortin receptor agonist reduces spontaneous and scheduled meal size but does not augment duodenal preload-induced feeding inhibition. Physiol Behav 2002;77:411-16.
32 Ste Marie L, Miura G, Marsh D, et al. A metabolic defect promotes obesity in mice lacking melanocortin-4 receptors. Proc Natl Acad Sci USA 2000;97:12339-44.

33 Williams D, Kaplan J, Grill $H$. The role of the dorsal vagal complex and the vagus nerve in feeding effects of melanocortin-3/4 receptor stimulation. Endocrinology 2000;141:1332-7.

34 Valassi E, Scacchi M, Cavagnini F. Neuroendocrine control of food intake. Nutr Metab Cardiovasc Dis 2008;18:158-68.

35 Stice $\mathrm{E}, \mathrm{Spoor} \mathrm{S}, \mathrm{Ng} \mathrm{J}$, et al. Relation of obesity to consummatory and anticipatory food reward. Physiol Behav 2009;97:551-60.

36 DiLeone RJ, Taylor JR, Picciotto MR. The drive to eat: comparisons and distinctions between mechanisms of food reward and drug addiction. Nat Neurosci 2012:15:1330-5.

37 Aponte Y, Atasoy D, Sternson S. AGRP neurons are sufficient to orchestrate feeding behavior rapidly and without training. Nat Neurosci 2011:14:351-5.

38 Williams K, Elmquist J. Lighting up the hypothalamus: coordinated control of feeding behavior. Nat Neurosci 2011;14:277-8.

39 Page K, Chan 0, Arora J, et al. Effects of fructose vs glucose on regional cerebral blood flow in brain regions involved with appetite and reward pathways. JAMA 2013:309:63-70.

40 Page K, Arora J, Qiu M, et al. Small decrements in systemic glucose provoke increases in hypothalamic blood flow prior to the release of counterregulatory hormones. Diabetes 2009;58:448-52.

41 Monet $\mathrm{P}$, Franc J, Brasseur A, et al. [Arterial spin labeling: state of the art]. J Radiol 2009;90:1031-7

42 Paulsen S, Larsen L, Jelsing J, et al. Gene expression profiling of individua hypothalamic nuclei from single animals using laser capture microdissection and microarrays. J Neurosci Methods 2009;177:87-93.

43 Shin A, Berthoud H-R. Obesity surgery: happy with less or eternally hungry? Trends Endocrinol Metab 2013;24:101-8.

44 Müller M, Bosy-Westphal A, Heymsfield $\mathrm{S}$. Is there evidence for a set point that regulates human body weight? F1000 Med Rep 2010;2:59.

45 Stefater M, Pérez-Tilve D, Chambers A, et al. Sleeve gastrectomy induces loss of weight and fat mass in obese rats, but does not affect leptin sensitivity. Gastroenterology 2010;138:2426.

46 Nadreau E, Baraboi ED, Samson P, et al. Effects of the biliopancreatic diversion on energy balance in the rat. Int J Obes (Lond) 2006:30:419-29.

47 Warne J, Padilla B, Horneman $\mathrm{H}$, et al. Metabolic and neuroendocrine consequences of a duodenal-jejunal bypass in rats on a choice diet. Ann Surg 2009:249:269-76

48 Romanova I, Ramos E, Xu Y, et al. Neurobiologic changes in the hypothalamus associated with weight loss after gastric bypass. J Am Coll Surg 2004;199:887-95

49 Fry M, Hoyda TD, Ferguson AV. Making sense of it: roles of the sensory circumventricular organs in feeding and regulation of energy homeostasis. Exp Biol Med (Maywood) 2007:232:14-26.

50 Halatchev I, Cone R. Peripheral administration of PYY(3-36) produces conditioned taste aversion in mice. Cell Metab 2005:1:159-68.

51 Camilleri M, Toouli J, Herrera M, et al. Intra-abdominal vagal blocking (VBLOC therapy): clinical results with a new implantable medical device. Surgery 2008:143:723-31.

52 Browning KN, Fortna SR, Hajnal A. Roux-en-Y gastric bypass reverses the effects of diet-induced obesity to inhibit the responsiveness of central vagal motoneurones. J Physiol 2013;591:2357-72.

53 Nestoridi E, Kvas S, Kucharczyk J, et al. Resting energy expenditure and energetic cost of feeding are augmented after Roux-en-Y gastric bypass in obese mice. Endocrinology 2012:153:2234-44.

54 Ochner CN, Kwok Y, Conceicao E, et al. Selective reduction in neural responses to high calorie foods following gastric bypass surgery. Ann Surg 2011; 253:502-7.

55 Thaler JP, Cummings DE. Minireview: Hormonal and metabolic mechanisms of diabetes remission after gastrointestinal surgery. Endocrinology 2009;150:2518-25.

56 Phillips RJ, Powley TL. Gastric volume rather than nutrient content inhibits food intake. Am J Physiol 1996;271:R766-9.

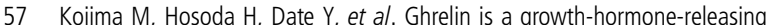
acylated peptide from stomach. Nature 1999;402:656-60.

58 Delhanty P, Neggers S, van der Lely A. Mechanisms in endocrinology: Ghrelin: the differences between acyl- and des-acyl ghrelin. Eur J Endocrino/2012;167:601-8.

59 Cummings DE, Weigle DS, Frayo RS, et al. Plasma ghrelin levels after diet-induced weight loss or gastric bypass surgery. N Engl J Med 2002;346:1623-30.

60 Fruhbeck G, Diez Caballero A, Gil MJ. Fundus functionality and ghrelin concentrations after bariatric surgery. N Engl J Med 2004;350:308-9.

61 Silk DB, Webb JP, Lane AE, et al. Functional differentiation of human jejunum and ileum: a comparison of the handling of glucose, peptides, and amino acids. Gut 1974:15:444-9.

62 Wang $P$, Caspi L, Lam C, et al. Upper intestinal lipids trigger a gut-brain-liver axis to regulate glucose production. Nature 2008:452:1012-16. 
63 Rubino F, Forgione A, Cummings DE, et al. The mechanism of diabetes control after gastrointestinal bypass surgery reveals a role of the proximal small intestine in the pathophysiology of type 2 diabetes. Ann Surg 2006:244:741-9.

64 Klein S, Fabbrini E, Patterson BW, et al. Moderate effect of duodenal-jejunal bypass surgery on glucose homeostasis in patients with type 2 diabetes. Obesity 2012;20:1266-72.

65 Geloneze B, Geloneze SR, Fiori C, et al. Surgery for nonobese type 2 diabetic patients: an interventional study with duodenal-jejunal exclusion. Obes Surg 2009:19:1077-83.

66 Bradley D, Magkos F, Klein S. Effects of bariatric surgery on glucose homeostasis and type 2 diabetes. Gastroenterology 2012;143:897-912.

67 Schauer PR, Kashyap SR, Wolski K, et al. Bariatric surgery versus intensive medical therapy in obese patients with diabetes. N Engl J Med 2012;366:1567-76.

68 Peterli R, Wölnerhanssen B, Peters T, et al. Improvement in glucose metabolism after bariatric surgery: comparison of laparoscopic Roux-en-Y gastric bypass and laparoscopic sleeve gastrectomy: a prospective randomized trial. Ann Surg 2009;250:234-41.

69 Lee W-J, Chong K, Ser K-H, et al. Gastric bypass vs sleeve gastrectomy for type 2 diabetes mellitus: a randomized controlled trial. Arch Surg 2011;146:143-8.

70 Shah P, Todkar J, Shah S. Effectiveness of laparoscopic sleeve gastrectomy on glycemic control in obese Indians with type 2 diabetes mellitus. Surg Obes Relat Dis 2010:6:138-41.

71 Gill R, Birch D, Shi $X$, et al. Sleeve gastrectomy and type 2 diabetes mellitus: a systematic review. Surg Obes Relat Dis 2010;6:707-13.

72 Li P, Fu P, Chen J, et al. Laparoscopic Roux-en-Y gastric bypass vs. laparoscopic sleeve gastrectomy for morbid obesity and diabetes mellitus: a meta-analysis of sixteen recent studies. Hepatogastroenterology 2013;60:132-7.

73 Cummings DE, Overduin J. Gastrointestinal regulation of food intake. J Clin Invest 2007:117:13-23.

74 Jang HJ, Kokrashvili Z, Theodorakis MJ, et al. Gut-expressed gustducin and taste receptors regulate secretion of glucagon-like peptide-1. Proc Natl Acad Sci USA 2007; 104:15069-74.

75 Theodorakis MJ, Carlson O, Michopoulos S, et al. Human duodenal enteroendocrine cells: source of both incretin peptides, GLP-1 and GIP. Am J Physiol Endocrinol Metab 2006;290:E550-9.

76 Rogers G, Tolhurst G, Ramzan A, et al. Electrical activity-triggered glucagon-like peptide-1 secretion from primary murine L-cells. J Physiol 2011;589:1081-93.

77 Flint A, Raben A, Astrup A, et al. Glucagon-like peptide 1 promotes satiety and suppresses energy intake in humans. J Clin Invest 1998;101:515-20.

78 Orskov C, Holst J, Nielsen 0. Effect of truncated glucagon-like peptide-1 [proglucagon-(78-107) amide] on endocrine secretion from pig pancreas, antrum, and nonantral stomach. Endocrinology 1988;123:2009-13.

79 Marathe C, Rayner C, Jones K, et al. Glucagon-like peptides 1 and 2 in health and disease: a review. Peptides 2013;44:75-86.

80 Toft-Nielsen M, Damholt M, Madsbad S, et al. Determinants of the impaired secretion of glucagon-like peptide-1 in type 2 diabetic patients. J Clin Endocrinol Metab 2001;86:3717-23.

81 Muscelli E, Mari A, Casolaro A, et al. Separate impact of obesity and glucose tolerance on the incretin effect in normal subjects and type 2 diabetic patients. Diabetes 2008:57:1340-8.

82 Laferrere $B$, Heshka $S$, Wang $K$, et al. Incretin levels and effect are markedly enhanced 1 month after RouX-en- $Y$ gastric bypass surgery in obese patients with type 2 diabetes. Diabetes Care 2007;30:1709-16.

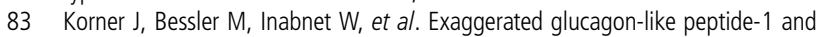
blunted glucose-dependent insulinotropic peptide secretion are associated with Roux-en-Y gastric bypass but not adjustable gastric banding. Surg Obes Relat Dis 2007:3:597-601.

84 Strader A, Clausen T, Goodin S, et al. Ileal interposition improves glucose tolerance in low dose streptozotocin-treated diabetic and euglycemic rats. Obes Surg 2009; 19:96-104.

85 Cummings B, Strader A, Stanhope $\mathrm{K}$, et al. lleal interposition surgery improves glucose and lipid metabolism and delays diabetes onset in the UCD-T2DM rat. Gastroenterology 2010;138:2437

86 Culnan $D$, Albaugh $V$, Sun $M$, et al. Ileal interposition improves glucose tolerance and insulin sensitivity in the obese Zucker rat. Am J Physiol Gastrointest Liver Physiol 2010:299:60

87 Shah M, Law JH, Micheletto F, et al. The contribution of endogenous glucagon-like peptide- 1 to glucose metabolism after Roux-en-Y gastric bypass. Diabetes Published Online First: 2 Oct 2013. doi: 10.2337/db13-0954

88 Astrup A, Rössner S, Van Gaal L, et al. Effects of liraglutide in the treatment of obesity: a randomised, double-blind, placebo-controlled study. Lancet 2009;374:1606-16.
89 Wilson-Perez HE, Chambers AP, Ryan KK, et al. Vertical sleeve gastrectomy is effective in two genetic mouse models of glucagon-like Peptide 1 receptor deficiency. Diabetes 2013:62:2380-5.

90 Batterham RL, Cohen MA, Ellis SM, et al. Inhibition of food intake in obese subjects by peptide YY3-36. N Engl J Med 2003;349:941-8.

91 Batterham RL, Cowley MA, Small CJ, et al. Gut hormone PYY(3-36) physiologically inhibits food intake. Nature 2002;418:650-4.

92 Yousseif A, Emmanuel J, Karra E, et al. Differential effects of laparoscopic sleeve gastrectomy and laparoscopic gastric bypass on appetite, circulating acyl-ghrelin, peptide YY3-36 and active GLP-1 levels in non-diabetic humans. Obes Surg Published Online First: 31 Aug 2013. doi: 10.1007/s11695013-1066-0

93 Gantz I, Erondu N, Mallick M, et al. Efficacy and safety of intranasal peptide YY3-36 for weight reduction in obese adults. J Clin Endocrinol Metab 2007;

92:1754-7.

94 Witte AB, Gryback P, Holst J, et al. Differential effect of PYY1-36 and PYY3-36 on gastric emptying in man. Regul Pept 2009;158:57-62.

95 Nightingale JM, Kamm MA, van der Sijp JR, et al. Gastrointestinal hormones in short bowel syndrome. Peptide YY may be the 'colonic brake' to gastric emptying. Gut 1996;39:267-72.

96 Allen JM, Fitzpatrick ML, Yeats JC, et al. Effects of peptide $Y Y$ and neuropeptide $Y$ on gastric emptying in man. Digestion 1984;30:255-62.

97 Stylopoulos N, Hoppin A, Kaplan L. Roux-en-Y gastric bypass enhances energy expenditure and extends lifespan in diet-induced obese rats. Obesity (Silver Spring, Md) 2009;17:1839-47

98 Zechner J, Mirshahi U, Satapati S, et al. Weight-independent effects of roux-en-Y gastric bypass on glucose homeostasis via melanocortin-4 receptors in mice and humans. Gastroenterology 2013;144:580-90.

99 Sumithran P, Prendergast LA, Delbridge $E$, et al. Long-term persistence of hormonal adaptations to weight loss. N Engl J Med 2011;365:1597-604.

100 Turnbaugh PJ, Ley RE, Mahowald MA, et al. An obesity-associated gut microbiome with increased capacity for energy harvest. Nature 2006;444:1027-31.

101 Backhed F, Ding $H$, Wang $T$, et al. The gut microbiota as an environmental factor that regulates fat storage. Proc Natl Acad Sci USA 2004;101:15718-23.

102 Ley RE, Backhed F, Turnbaugh P, et al. Obesity alters gut microbial ecology. Proc Natl Acad Sci USA 2005;102:11070-5

103 Zhang $\mathrm{H}$, DiBaise JK, Zuccolo A, et al. Human gut microbiota in obesity and after gastric bypass. Proc Natl Acad Sci USA 2009;106:2365-70.

104 Li JV, Ashrafian H, Bueter M, et al. Metabolic surgery profoundly influences gut microbial-host metabolic cross-talk. Gut 2011;60:1214-23.

105 Liou AP, Paziuk M, Luevano JM Jr, et al. Conserved shifts in the gut microbiota due to gastric bypass reduce host weight and adiposity. Sci Trans/ Med 2013;5:178ra41.

106 Aron-Wisnewsky J, Dore J, Clement K. The importance of the gut microbiota after bariatric surgery. Nat Rev Gastroenterol Hepatol 2012;9:590-8.

107 Abu Dayyeh BK, Thompson CC. Obesity and bariatrics for the endoscopist: new techniques. Therap Adv Gastroenterol 2011;4:433-42.

108 Gersin KS, Rothstein RI, Rosenthal RJ, et al. Open-label, sham-controlled trial of an endoscopic duodenojejunal bypass liner for preoperative weight loss in bariatric surgery candidates. Gastrointest Endosc 2010;71:976-82

109 Schouten R, Rijs CS, Bouvy ND, et al. A multicenter, randomized efficacy study of the EndoBarrier Gastrointestinal Liner for presurgical weight loss prior to bariatric surgery. Ann Surg 2010;251:236-43.

110 Cohen RV, Neto MG, Correa JL, et al. A pilot study of the duodenal-jejunal bypass liner in low body mass index type 2 diabetes. J Clin Endocrinol Metab 2013;98: E279-82.

111 de Jonge C, Rensen SS, Koek GH, et al. Endoscopic Duodenal-Jejunal Bypass Liner Rapidly Improves Plasma Parameters of Nonalcoholic Fatty Liver Disease. Clin Gastroenterol Hepatol 2013;11:1517-20.

112 de Jonge C, Rensen SS, Verdam FJ, et al. Endoscopic duodenal-jejunal bypass line rapidly improves type 2 diabetes. Obes Surg 2013;23:1354-60.

113 Habegger KM, Al-Massadi 0, Heppner KM, et al. Duodenal nutrient exclusion improves metabolic syndrome and stimulates villus hyperplasia. Gut Published Online First: 9 Oct 2013. doi: 10.1136/gutjnl-2013-304583

114 Cohen R, le Roux CW, Papamargaritis D, et al. Role of proximal gut exclusion from food on glucose homeostasis in patients with Type 2 diabetes. Diabet Med 2013;30:1482-6.

115 Abu Dayyeh B, Rajan E, Gostout C. Endoscopic sleeve gastroplasty: a potential endoscopic alternative to surgical sleeve gastrectomy for treatment of obesity. Gastrointest Endosc 2013;78:530-5. 